\title{
Gravity field determination of a Comet Nucleus: Rosetta at P/Wirtanen
}

\author{
M. Pätzold ${ }^{1}$, B. Häusler ${ }^{2}$, A. Wennmacher ${ }^{1}$, K. Aksnes ${ }^{3}$, J. D. Anderson ${ }^{4}$, S. W. Asmar ${ }^{4}$, J.-P. Barriot ${ }^{5}$, \\ H. Boehnhardt ${ }^{6}$, W. Eidel ${ }^{2}$, F. M. Neubauer ${ }^{1}$, O. Olsen ${ }^{3}$, J. Schmitt ${ }^{1}$, J. Schwinger ${ }^{1}$, and N. Thomas ${ }^{7}$ \\ 1 Institut für Geophysik und Meteorologie, Universität zu Köln, Albertus-Magnus-Platz, 50923 Köln, Germany \\ e-mail: wennmach@geo.Uni-Koeln.DE \\ 2 Institut für Raumfahrttechnik, Universität der Bundeswehr München, 85557 Neubiberg, Germany \\ e-mail: Bernd.Haeusler@UniBW-Muenchen.DE; werner.eidel@UniBW-Muenchen.DE \\ 3 Institute for Theoretical Astrophysics, University of Oslo, Oslo, Norway \\ e-mail: Kaare.Aksnes@uio.no; Oystein.Olsen@uio.no \\ 4 Jet Propulsion Laboratory, Caltech, 4800 Oak Grove drive, Pasadena, CA 91107, USA \\ e-mail: John.D.Anderson@jpl.nasa.gov; Sami.W.Asmar@jpl.nasa.gov \\ 5 Observatoire Midi-Pyrénées, Toulouse, France \\ e-mail: jean-pierre.barriot@cnes.fr \\ 6 European Southern Observatory, Santiago de Chile, Chile \\ e-mail: hboehnha@eso.org \\ 7 Max-Planck-Institut für Aeronomie, 37189 Katlenburg-Lindau, Germany \\ e-mail: thomas@linmpi.mpg.de
}

Received 18 May 2001 / Accepted 7 June 2001

\begin{abstract}
One of the prime objectives of the Rosetta Radio Science Investigations (RSI) experiment is the determination of the mass, the bulk density and the low degree and order gravity of the nucleus of comet $\mathrm{P} /$ Wirtanen, the target object of the international Rosetta mission. The RSI experiment will use the spacecraft's radio carrier frequencies at $X$-band $(8.4 \mathrm{GHz})$ and $S$-band $(2.3 \mathrm{GHz})$ in order to measure slight changes of the orbit velocity via the classical Doppler effect induced by the gravity attraction of the comet nucleus. Based on an estimate of the background Doppler noise, it is expected that a mass determination (assuming a representative radius of $700 \mathrm{~m}$ and a bulk density of $500 \mathrm{~kg} / \mathrm{m}^{3}$ ) at an accuracy of $0.1 \%$ can be achieved if the spacecraft's orbit is iteratively reduced below $7 \mathrm{~km}$ altitude. The gravity field of degree and order two can be detected for reasonable tracking times below $5 \mathrm{~km}$ altitude. The major competing forces acting on the spacecraft are the radiation pressure and the gas mass flux from cometary activity. While the radiation pressure may be predicted, it is recommended to begin a gravity mapping campaign well before the onset of outgassing activity (>3.25 AU heliocentric distance). Radial acceleration by water outgassing is larger by orders of magnitude than the accelerations from the low degree and order gravity field and will mask the contributions from the gravity field.
\end{abstract}

Key words. celestial mechanics - space vehicles: Rosetta - comets: individual: 46 P/Wirtanen

\section{Introduction}

The Rosetta spacecraft will be launched in January 2003 to intercept comet $\mathrm{P} /$ Wirtanen in 2011 and to escort the comet in an orbit about its nucleus from Rendezvous (about one year after the comet's aphelion) to its perihelion in July 2013. The Rosetta Radio Science Investigations (RSI) experiment onboard of Rosetta (Pätzold et al. 2001) will focus in the early part of the

Send offprint requests to: M. Pätzold,

e-mail: paetzold@geo.Uni-Koeln.DE prime mission on the determination of the mass, bulk density and the low order gravity field of the comet nucleus.

RSI will use the two-way radio carrier signals at $X$ band uplink $(7168 \mathrm{MHz})$ and $S$-band $(2300 \mathrm{MHz})$ and $X$-band $(8422 \mathrm{MHz})$ simultaneous downlink, provided by the spacecraft radio subsystem, in order to measure slight changes in the spacecraft velocity induced by the attraction of the asymmetric nucleus gravity field via the classical Doppler effect. If the spacecraft's velocity is changed by forces acting on the spacecraft, a frequency shift of the radio carrier with respect to a predicted Doppler shift 
(from the heliocentric motion of the nucleus as a point mass relative to the ground station) is induced by

$\Delta f=-\frac{f_{0}}{c} \cdot \frac{\Delta R}{\Delta t}$

where $\Delta R$ is the change of the distance between the spacecraft and the ground station (topocenter) within the integration time $\Delta t$ caused by non-accounted forces acting on the spacecraft, $f_{0}$ is the radio carrier frequency at $X$ band and $c$ is the speed of light. If the two-way radio link is used as described above, the Doppler shift is twice the value of (1).

Determination of the cometary mass and bulk density is a fundamental objective required to assess the validity and accuracy of various cometary models. Extensive simulation studies, in preparation for the Near Earth Asteroid Rendezvous (NEAR) mission to asteroid 433 Eros, demonstrate that orbiting a small body will require a gravity field extraction process fundamentally different from previous gravity studies of the planets or moons (Scheeres 1995; Miller et al. 1995). Upon arrival at the comet, the nucleus size, shape, mass, activity and spin state will be poorly known. $\mathrm{P} /$ Wirtanen is significantly smaller than asteroid 433 Eros and will most likely be active during some portions of the gravity field investigation campaign (Drechsel et al. 2000). A strategy is needed for an iterative solution of the gravity field.

\section{The size of the nucleus and Spacecraft orbits about the nucleus}

The estimates of the nucleus size for comet $\mathrm{P} /$ Wirtanen vary considerably. While Boehnhardt et al. (1997) estimate a nucleus radius $R_{\mathrm{c}}$ of $700 \mathrm{~m}$ to $800 \mathrm{~m}$ from ground-based observations, a radius $R_{\mathrm{c}}$ of $600 \mathrm{~m}$ was reported by Lamy et al. (1998) from HST observations. Observations of $\mathrm{P} /$ Wirtanen during the recent aphelion passage revealed a radius $R_{\mathrm{c}}$ of $550 \pm 40 \mathrm{~m}$ (Boehnhardt et al. 2001) in agreement with Lamy et al. (1987). An overview of the Wirtanen observations is given by Schulz \& Schwehm (1999).

Very little is known about Wirtanen's shape. Lamy et al. (1998) derive a ratio of $a / b>1.2$ from the light curve, consistent with Boehnhardt et al. (2001) new observations of $a / b>1.4$. However, taking the nucleus of $\mathrm{P} /$ Halley (Keller et al. 1986) or the shapes of known asteroids like Gaspra and Ida or the Martian moons Phobos and Deimos as examples, it is likely that the shape of the $\mathrm{P} /$ Wirtanen nucleus is a tri-axial ellipsoid with axis ratio close to 2:1:1.

Estimates of the bulk density of cometary nuclei are also varying in wide ranges from $200 \mathrm{~kg} / \mathrm{m}^{3}$ to $1000 \mathrm{~kg} / \mathrm{m}^{3}$ (e.g. Greenberg \& Hage 1990; Rickman 1987; Rickman et al. 1989; Sekanina \& Yeomans 1985). The determination of the bulk density is one fundamental property in models for cometary nuclei and one of the prime objectives of the Rosetta RSI experiment (Pätzold et al. 2001). The orbital velocity and the perturbation of a spacecraft orbiting a non-active comet nucleus is then defined by the size, shape and mass of the nucleus (gravitational attraction).

In the following, we shall investigate three nucleus mass models, two extreme cases and one average model:

1. a large radius $R_{\mathrm{c}}$ of $1000 \mathrm{~m}$ and a high bulk density of $1000 \mathrm{~kg} / \mathrm{m}^{3}$ (the "wishfull thinking" case);

2. a small radius $R_{\mathrm{c}}$ of $500 \mathrm{~m}$ and a low bulk density of $200 \mathrm{~kg} / \mathrm{m}^{3}$ (the "nightmare" case);

3. an average model of a radius $R_{\mathrm{c}}$ of $700 \mathrm{~m}$ according to the observations and an average bulk density of $500 \mathrm{~kg} / \mathrm{m}^{3}$.

Assuming for simplicity a spherical body, the orbital velocity of a spacecraft in a circular orbit about that body is essentially in the order of decimeter per second (Fig. 1, upper panel). Figure 1, lower panel, is the spacecraft's orbital revolution period $P$. At $10 \mathrm{~km}$ distance to the center of mass of the nucleus the revolution period is in the order of several 100 hours.

\section{Determination of GM}

\subsection{Accuracy estimates}

Taking all major instrumental noise sources into account (spacecraft radio subsystem and ground station), the Doppler velocity error $\sigma_{\mathrm{v}_{0}}$ at $S$-band and $X$-band for the Rosetta transponder system was estimated for a one second integration time (Table 1). For longer integrations, the Doppler velocity error $\sigma_{0}(\Delta t)$ scales with the square root of the integration time $\Delta t$ and is shown in Fig. 2:

$\sigma_{\mathrm{v}}(\Delta t)=\sigma_{\mathrm{v}_{0}} \frac{1}{\sqrt{\Delta t}}$.

Another uncertainty which adds to the Doppler noise is the propagation of the radio signals through the turbulent solar wind (Bird 1982; Pätzold et al. 2001). Turbulent solar wind density variations along the radio ray path will induce dispersive phase shifts on the radio carrier, increasing the phase noise. Using a dual-frequency downlink at $S$-band and $X$-band, it is possible to limit the solar wind noise by extracting the dispersive propagation contributions from the calculation of the differential Doppler and by correcting the $X$-band two-way radio link by this result.

At the time of the projected RSI gravity campaign (June to July 2012) the comet is at a heliocentric distance of 3.25 AU and at a geocentric distance of 2.5 AU (Fig. 3) and fortunately at solar opposition. Therefore contributions from the turbulent solar wind to dispersive phase shifts are minimized but cannot be neglected.

The sphere of gravitational influence (Hill sphere) about the cometary nucleus is estimated from

$r_{\text {Hill }}=R\left(\frac{1}{3} \frac{M}{M_{\odot}}\right)^{\frac{1}{3}}$

where $R$ is the heliocentric distance, $M$ and $M_{\odot}$ are the mass of the comet and of the Sun, respectively. 

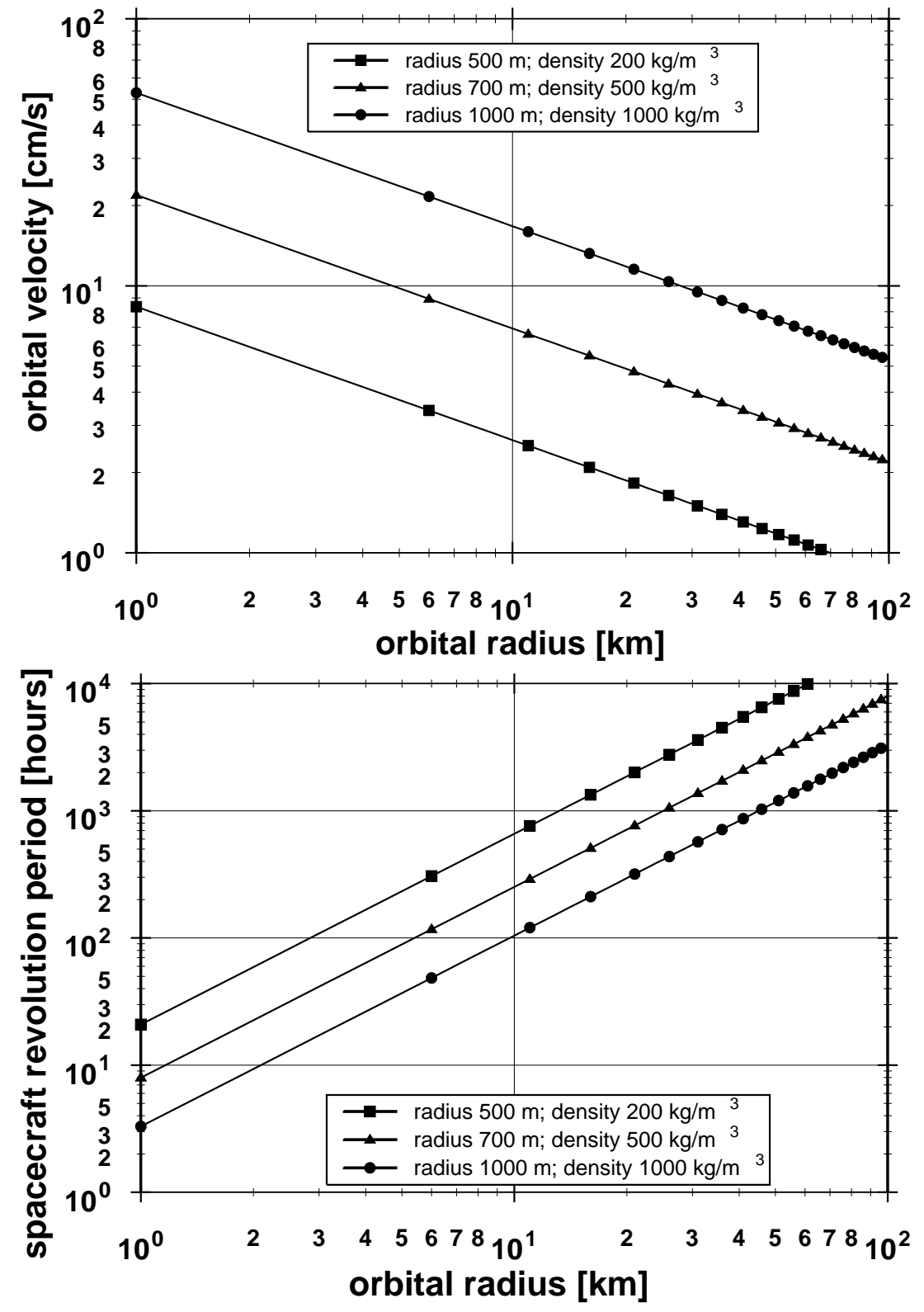

Fig. 1. Three different nucleus mass models are considered in the following Figures: a comet nucleus having a large radius of $1000 \mathrm{~m}$ and a high bulk density of $1000 \mathrm{~kg} / \mathrm{m}^{3}$ (circles), a small radius of $500 \mathrm{~m}$ and a low bulk density of $200 \mathrm{~kg} / \mathrm{m}^{3}$ (squares) and an average mass model of a nucleus of $700 \mathrm{~m}$ radius and a bulk density of $500 \mathrm{~kg} / \mathrm{m}^{3}$ (triangles). The upper panel shows the spacecraft orbital velocity (assuming a circular orbit for simplicity) as a function of orbital radius or distance to the nucleus. At $5 \mathrm{~km}$ distance to the nucleus the velocity is in the order of $10 \mathrm{~cm} / \mathrm{s}$ for the average nucleus mass model. The lower panel shows the revolution period of the spacecraft about the nucleus. At the same distance of $5 \mathrm{~km}$ the revolution period is 100 hours for the average mass model.

Total Doppler Noise

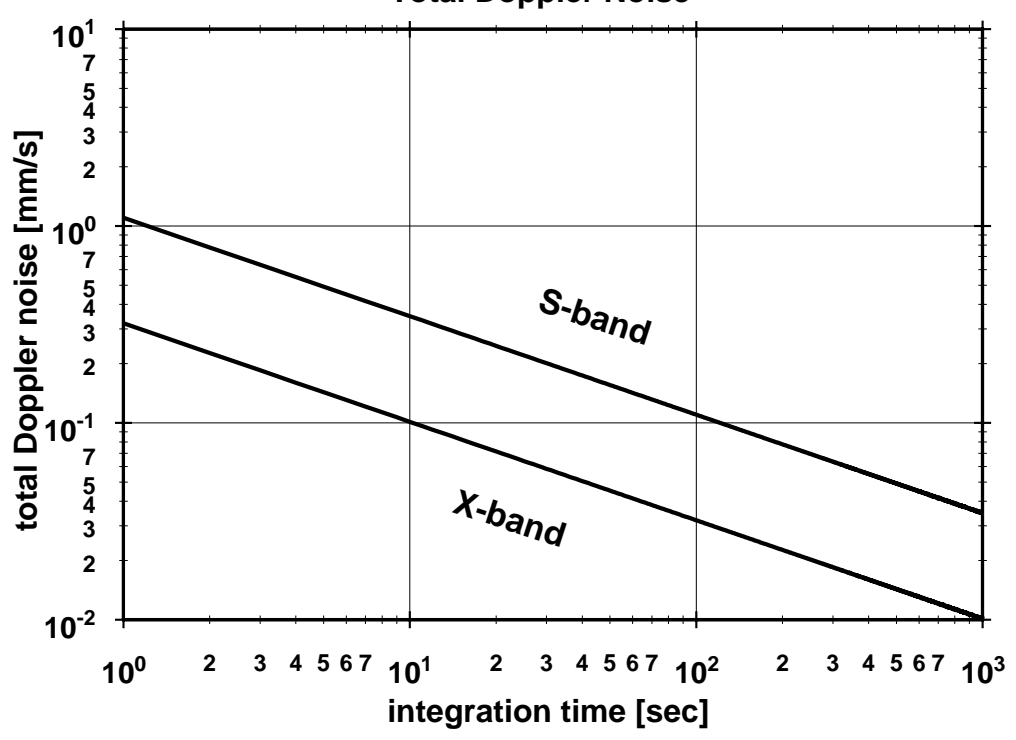

Fig. 2. Estimated instrumental Doppler noise (from the radio subsystem and the ground station) at $S$-band (upper curve) and $X$-band (lower curve) as a function of integration time. Integration times of $>100 \mathrm{~s}$ are planned to be used for gravity investigations. 


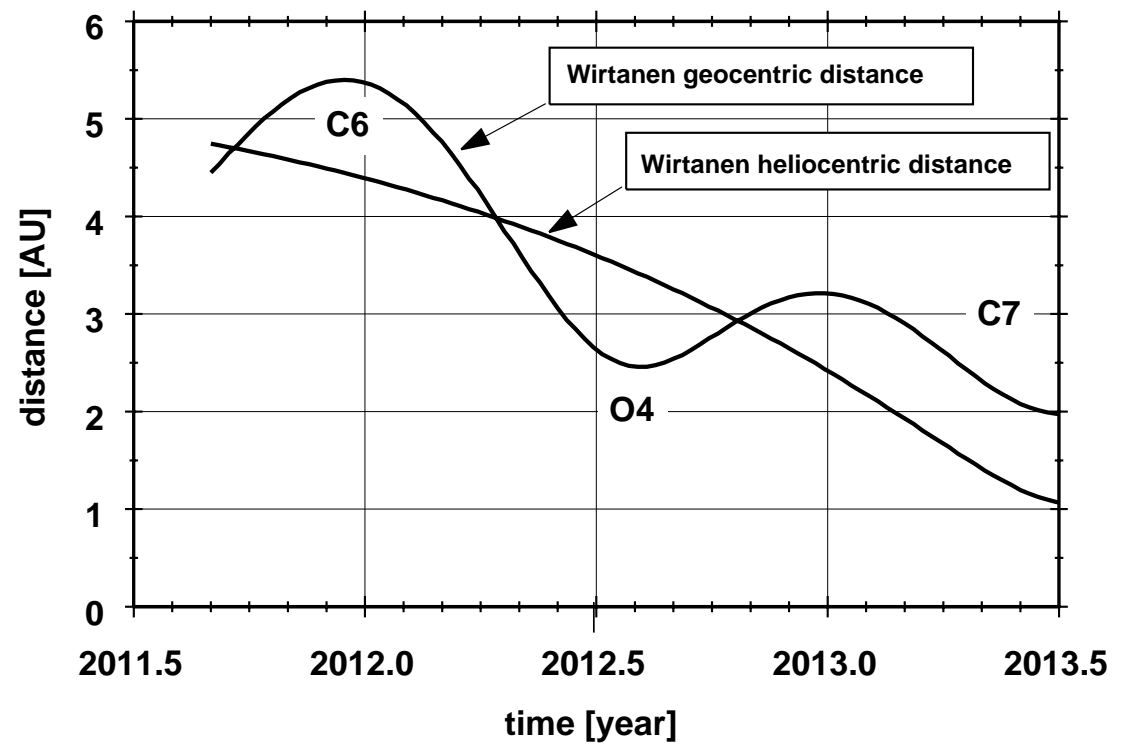

Fig. 3. Heliocentric distance and geocentric distance of comet $\mathrm{P} /$ Wirtanen from September 2011 (Rosetta arrival) to July 2013 (perihelion). The geocentric distance is instrumental for the signal-to-noise ratio of the received radio signal transmitted by spacecraft. C6 and C7 mark the sixth and the seventh solar conjunction, respectively, since the launch (spacecraft is behind the Sun as seen from Earth). During a solar conjunction, the radio signal propagates through the dense and turbulent plasma region of the solar corona leading to an increased phase noise of the radio carrier signals. O4 mark the fourth solar opposition since launch. The solar wind phase noise contributions are minimized during a solar opposition and this is the preferred time period to perform the nucleus gravity investigations. The comet itself is probably not very active between $3.5 \mathrm{AU}$ and 3.25 AU heliocentric distance.
The Hill sphere radius is $300 \mathrm{~km}$ at the heliocentric approach distance of approximately $4 \mathrm{AU}$ for the average nucleus mass model (Fig. 4).

Injection into a high bound orbit should enable a mass determination to an accuracy of $10 \%$ at a distance within $60 \mathrm{~km}$ (Fig. 5) for the average Wirtanen mass model. This accuracy allows the iterative reduction of the orbit radius and improvements of the mass determination. Assuming a circular orbit with radius $r$, the gravity acceleration is defined as

$a(r)=\frac{G M}{r^{2}}$

where $G=6.672 \times 10^{-11} \mathrm{~m}^{3} \mathrm{~kg}^{-1} \mathrm{~s}^{-2}$ is the gravitational constant. It follows for the fractional $G M$ error for a tracking pass of length $T$

$\frac{\sigma_{G M}}{G M}=\frac{r^{2}}{G M} \frac{1}{T} \sigma_{\mathrm{v}}(\Delta t)$.

An $1 \%$ error can be achieved if the orbit radius is within $8 \mathrm{~km}$ for a low density/small size comet (upper curve in Fig. 5 and also the lower limit for the Wirtanen mass models) and within $60 \mathrm{~km}$ for a high density/large size comet (lower curve in Fig. 5 and upper limit for the Wirtanen mass models).

Later orbit radius reduction allows iterative improvements of the mass determination down to the $0.1 \%$ level (Fig. 5) and yields distances between $3 \mathrm{~km}$ and $18 \mathrm{~km}$ for the two extreme Wirtanen mass models.

\section{Low degree and order gravity field}

Usually, the gravitational potential $U$ of a body is expanded into an infinite series of spherical harmonics of
Table 1. Doppler velocity error budget.

\begin{tabular}{lcc}
\hline \hline & \multicolumn{2}{c}{ Doppler velocity error $\sigma_{\mathrm{v}}$} \\
& $S$-band & $X$-band \\
\hline \hline Phase error & $1.0 \mathrm{~mm} / \mathrm{s}$ & $0.3 \mathrm{~mm} / \mathrm{s}$ \\
(thermal and ground & & \\
station contribution) & & \\
Transponder quantisation & $0.4 \mathrm{~mm} / \mathrm{s}$ & $0.1 \mathrm{~mm} / \mathrm{s}$ \\
error in frequency & & \\
Transponder quantisation & $0.01 \mathrm{~mm} / \mathrm{s}$ & $0.004 \mathrm{~mm} / \mathrm{s}$ \\
error in phase & & \\
\hline Total error (coherent mode) & $1.08 \mathrm{~mm} / \mathrm{s}$ & $0.32 \mathrm{~mm} / \mathrm{s}$ \\
\hline \hline
\end{tabular}

the form

$$
\begin{aligned}
U(r, \theta, \phi)= & -\frac{G M}{r}\left[1+\sum_{l=2}^{\infty} \sum_{m=0}^{l}\left(\frac{a}{r}\right)^{l} \bar{P}_{l m}(\cos \theta)\right. \\
& \left.\left\{\bar{C}_{l m} \cos m \phi+\bar{S}_{l m} \sin m \phi\right\}\right]
\end{aligned}
$$

where $r, \theta, \phi$ are the polar coordinates radius, colatitude and longitude, respectively. The $P_{l m}$ are the fully normalized associated Legendre polynominals of degree $l$ and order $m$. The fully normalized expansion coefficients are 


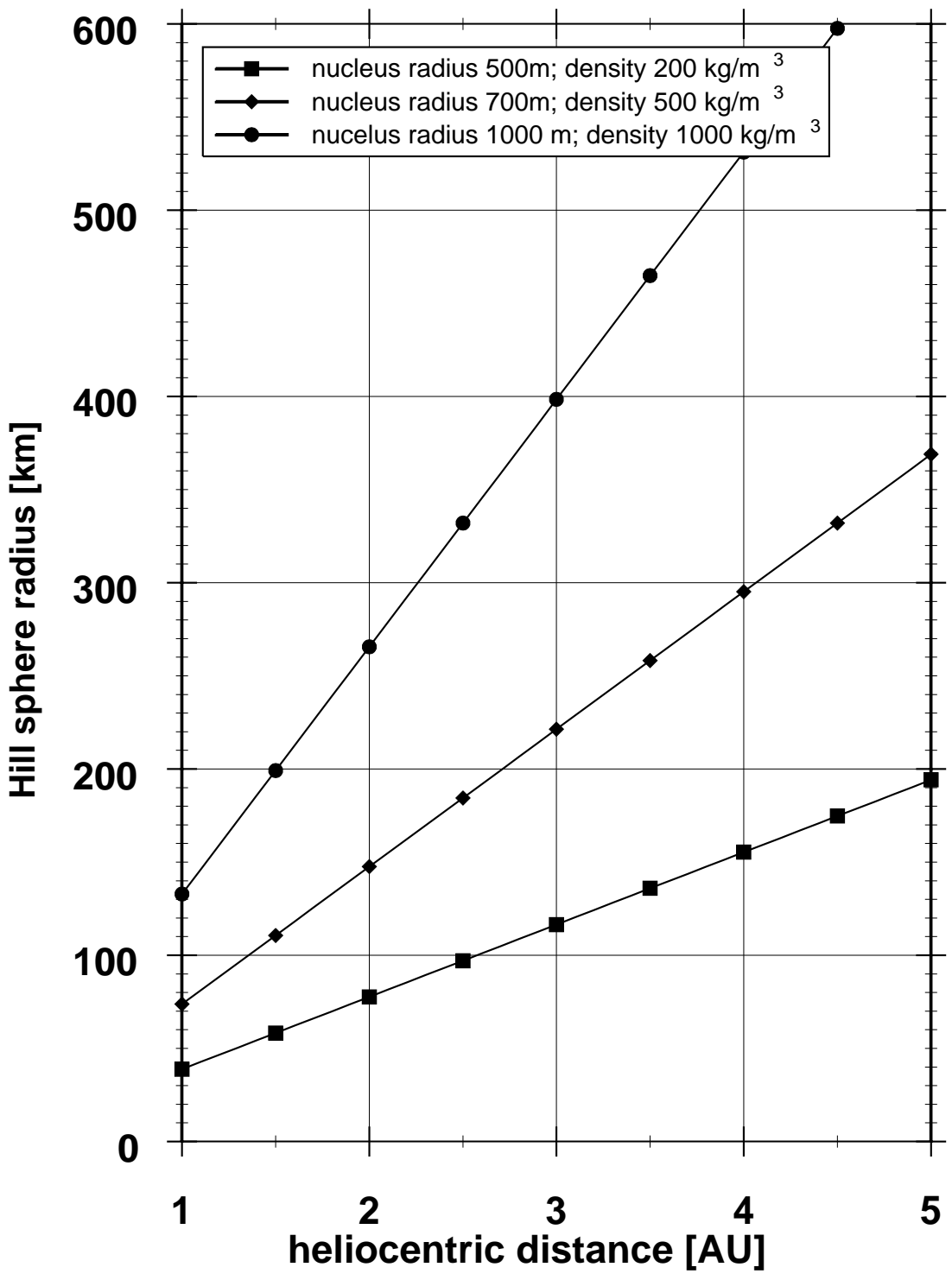

Fig. 4. Hill sphere radius or distance of gravitational influence for the three nucleus mass models. The radius is $250 \mathrm{~km}$ at $3.5 \mathrm{AU}$ heliocentric distance for the average mass model. represented by

$$
\begin{aligned}
& \left.\begin{array}{c}
\bar{C}_{l m} \\
\bar{S}_{l m}
\end{array}\right\}=\frac{1}{a^{l} M} \sqrt{\frac{2-\delta_{0 m}(l-m) !}{(2 l+1)(l+m) !}} \int_{0}^{a} \int_{0}^{\pi} \int_{0}^{2 \pi} \rho\left(r^{\prime}, \theta^{\prime}, \phi^{\prime}\right) . \\
& P_{l m}\left(\cos \theta^{\prime}\right)\left\{\begin{array}{c}
\cos m \phi^{\prime} \\
\sin m \phi^{\prime}
\end{array}\right\} r^{\prime l+2} \mathrm{~d} r^{\prime} \sin \theta^{\prime} \mathrm{d} \theta^{\prime} \mathrm{d} \phi^{\prime}
\end{aligned}
$$

where $\rho(r, \theta, \phi)$ is the density distribution of the nucleus. Because the higher harmonics fall off with distance as $r^{-(l+1)}$ only contributions from degree and order two are expected. Assuming that the center of mass coincides with the center of figure (no terms of degree and order one), and that the $z$-axis is aligned with the principal axis of the main moment of inertia, the reduced gravity potential is then

$$
\begin{aligned}
U(r, \theta, \phi)= & -\frac{G M}{r}\left[1+\left(\frac{a}{r}\right)^{2} \bar{P}_{20}(\cos \theta) \bar{C}_{20}\right. \\
& \left.+\left(\frac{a}{r}\right)^{2} \bar{P}_{22}(\cos \theta) \bar{C}_{22} \cos 2 \phi\right]
\end{aligned}
$$

$\bar{P}_{20}$ and $\bar{P}_{22}$ are the fully normalized associated Legendre polynominals of degree and order two.

For a first approximation, the gravity coefficients of second degree and order $\left(C_{20}\right.$ and $\left.C_{22}\right)$ will be estimated using the shape model (from imaging observations) and assuming constant density $\rho(r, \theta, \phi)=$ const. The result is a constraint on the true low degree and order gravity field. The coefficients $C_{20}$ and $C_{22}$ represents the gravitational oblateness and the equatorial ellipticity, respectively, of the body.

Assuming a tri-axial ellipsoid with equatorial radii $a$ and $b$ and polar radius $c$, a constant bulk density of the nucleus and choosing the equatorial radius $a$ as the reference radius, the second degree and order gravity coefficients (constant density) are expressed as

$$
\begin{aligned}
& C_{20}=\frac{1}{5 a^{2}}\left\{c^{2}-\frac{a^{2}+b^{2}}{2}\right\} \\
& C_{22}=\frac{1}{5 a^{2}}\left\{\frac{a^{2}-b^{2}}{4}\right\} .
\end{aligned}
$$




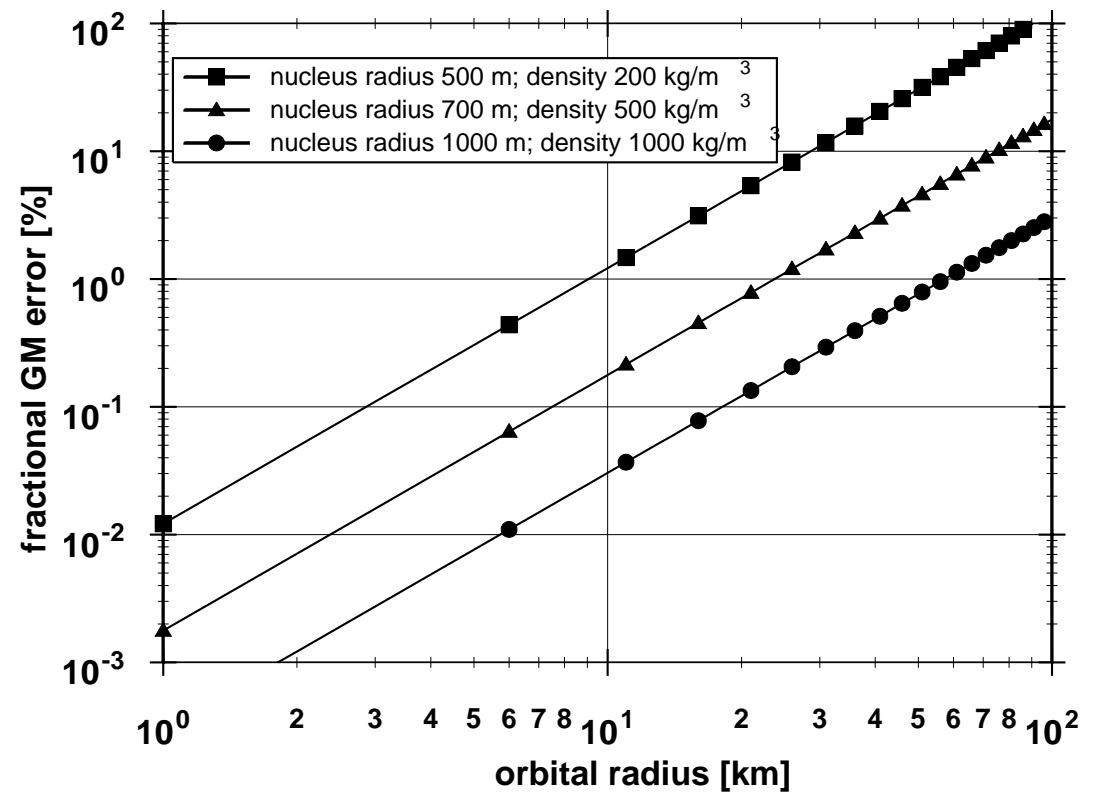

Fig. 5. Fractional GM (gravitational constant $\times$ mass) error as a function of spacecraft orbital radius. A $10 \%$ uncertainty can be achieved if the orbital radius is within $70 \mathrm{~km}$ for the average nucleus mass model. Then, the distance can iteratively reduced to lower orbits. A $0.1 \%$ error can be achieved if the spacecraft orbits within $7 \mathrm{~km}$ distance to the nucleus, a value required for the Rosetta lander descent trajectory.
Table 2 lists then, the numerical values of $C_{20}$ and $C_{22}$ for various axes ratios $a: b: c$.

For the average mass model with $R_{\mathrm{c}}=700 \mathrm{~m}$ and $a: b: c=2: 1: 1$, the equatorial radii are $a=1120 \mathrm{~m}$ and $b=560 \mathrm{~m}$ and the polar radius is $c=560 \mathrm{~m}$.

The resulting perturbing radial acceleration amplitudes for the fully normalized spherical harmonics and gravity coefficients of degree and order two are (Milani et al. 1987)

$$
\begin{aligned}
& \left|\frac{\partial U_{20}}{\partial r}\right|=\left|a_{20}\right|=3 \frac{G M}{r^{2}}\left(\frac{a}{r}\right)^{2} C_{20} \\
& \left|\frac{\partial U_{20}}{\partial r}\right|=\left|a_{22}\right|=3 \frac{G M}{r^{2}}\left(\frac{a}{r}\right)^{2} C_{22} .
\end{aligned}
$$

Figure 6 (left panel) shows the acceleration according to (10) as a function of orbital distance for our three Wirtanen mass models. The acceleration from the zero degree term $\left(G M / r^{2}\right)$ is also shown for comparison. At $r=5 \mathrm{~km}$ we find for the average mass model

$\left|a_{20}\right|=2.2 \times 10^{-8} \mathrm{~m} \mathrm{~s}^{-2}$
$\left|a_{22}\right|=1.1 \times 10^{-8} \mathrm{~m} \mathrm{~s}^{-2}$.

During the gravity mapping campaign, care should be taken in choosing orbits. As shown by Scheeres (1995) the effect of $C_{22}$ may cause the orbiter to either get ejected from a bound orbit or crash into the nucleus. Olsen (2000) showed that resonances may be employed to find stable orbits close to the nucleus. Using the perturbation method of Deprit (1969), the instantaneous coordinates and velocities of the stable orbits can be derived from the averaged equations.

In the likely event that the comet is not in perfect principal axis rotation, a knowledge of the body's secondorder gravity coefficients and a determination of its spin state can be used to determine its normalized moment of inertia

$I=\frac{C}{M a^{2}}$

which provides constraints upon the internal structure of the nucleus.

From an improved shape model (derived from imaging), the model can be refined and the gravity field can once again be approximated assuming a constant density nucleus. Mass will eventually be known to $0.1 \%$ (Fig. 5), volume and density better than $3 \%$. After eventually determining the true gravity coefficients, the shape gravity model (with constant density) and the true gravity model (from spacecraft tracking) can then be compared to yield information on the mass heterogeneity of the comet nucleus. This was done with the NEAR radio tracking data in an orbit about the asteroid 433 Eros. The comparison of the shape gravity model and the true gravity model revealed that both are equal to $1 \%$ and that Eros appears to have a uniform density distribution (Yeomans et al. 2000).

\section{Comparison with competing non-gravitational forces acting on the spacecraft}

The above strategy also assumes that cometary outgassing does not induce significant accelerations upon the spacecraft. Initial estimates have shown that an early activity of comet Wirtanen even beyond 3 AU, however, might generate radial accelerations due to outgassing that could mask the effects of higher gravity harmonics (Gill et al. 1996).

Assuming mass flux conservation, the gas density at distance $r$ from the comet is (Gill et al. 1996)

$\rho(r)=m_{\text {gas }} \frac{Q_{\text {gas }}}{4 \pi r^{2} v_{\text {gas }}}$ 


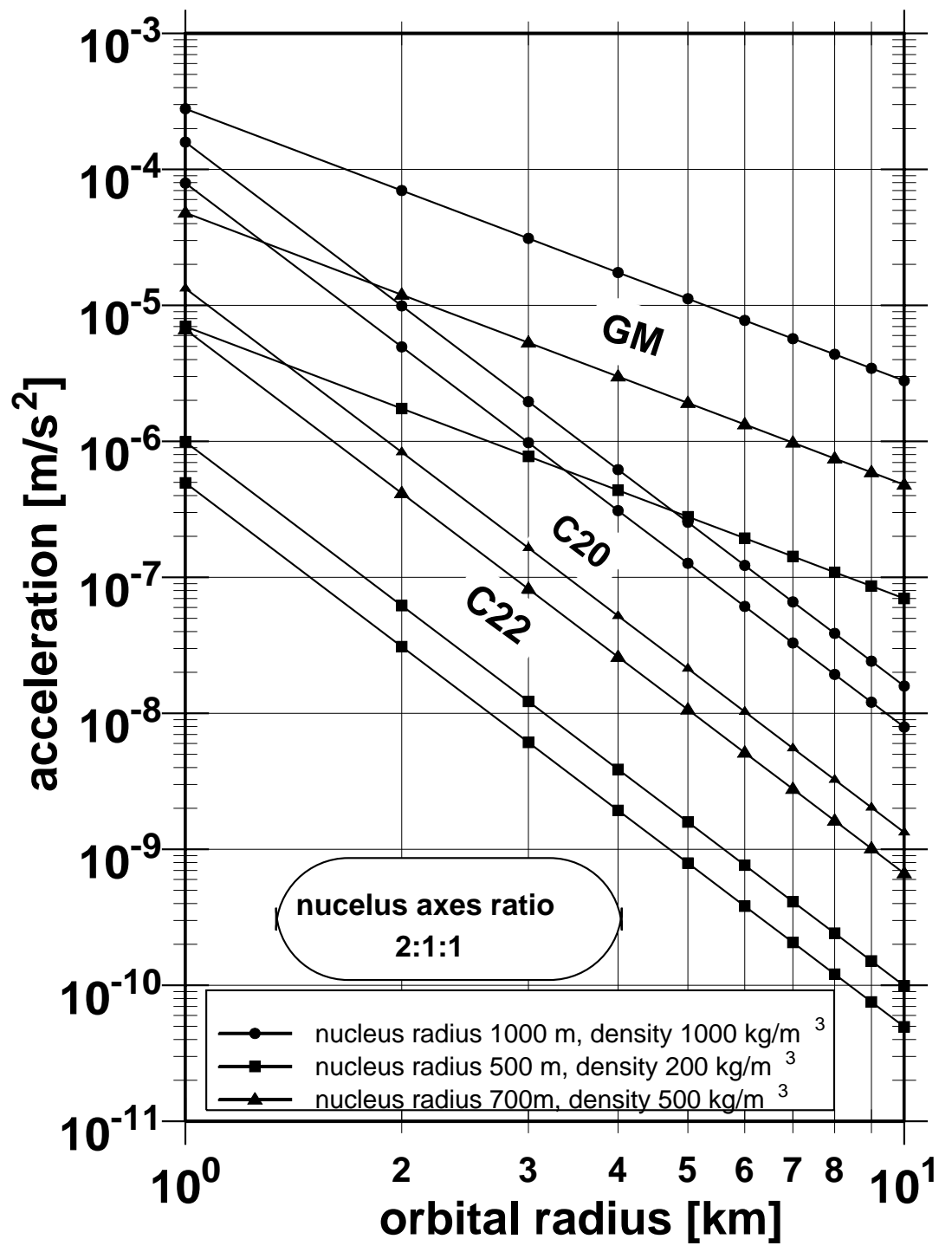

Fig. 6. Second degree and order gravity acceleration amplitudes $\left(C_{20}\right.$ and $C_{22}$ terms) as a function of orbital radius for the three nucleus mass models (left panel) having an axes ratio of $a: b: c=2: 1: 1$. Zero degree gravitational acceleration (point mass $G M)$ is also shown for comparison. The noise level at $600 \mathrm{~s}$ integration time is in the order of $10^{-9} \mathrm{~m} / \mathrm{s}^{2}$.
Table 2. Low degree and order two gravity coefficients, assuming constant bulk density.

\begin{tabular}{ccc}
\hline \hline $\begin{array}{c}\text { Axes ratio } \\
a: b: c\end{array}$ & $C_{20}$ & $C_{22}$ \\
\hline \hline $2: 1: 1$ & -0.075 & 0.038 \\
$2: 1.5: 1$ & -0.106 & 0.022 \\
$2: 1.8: 1.5$ & -0.069 & 0.0095 \\
$2: 2: 2$ & 0.000 & 0.000 \\
\hline \hline
\end{tabular}

where $m_{\text {gas }}$ is the mean molecular mass of the gas species, $Q_{\text {gas }}$ is the combined gas production rate, and $v_{\text {gas }}$ is the velocity of the gas molecules at distance $r$ from the nucleus.

Schulz \& Schwehm (1999) give the production rates for various gas molecules $\left(\mathrm{CN}, \mathrm{C}_{2}, \mathrm{C}_{3}, \mathrm{NH}_{2}\right)$ at approximately 3 AU. A combined production rate is then derived as $Q_{\text {gas }}<2.2 \times 10^{24} s^{-1}$ and the mean molecular mass of the above species is $3.87 \times 10^{-26} \mathrm{~kg}$. It follows a gas density of $\rho_{\text {gas }}<3.9 \times 10^{-13} \mathrm{~kg} / \mathrm{m}^{3}$ from (13) at a distance of $r=5 \mathrm{~km}$ from the nucleus assuming an outflow velocity of $v_{\text {gas }}=700 \mathrm{~m} / \mathrm{s}$ at that distance. However, gas velocities were observed as high as $1000 \mathrm{~m} / \mathrm{s}$ during the Giotto flyby at comet Halley (Krankowsky et al. 1986).

Stern et al. (1998) were not able to detect water at 2.7 AU from HST observations and concluded that $Q_{\mathrm{H}_{2} \mathrm{O}}<1.2 \times 10^{26} \mathrm{~s}^{-1}$. A water production rate of $Q_{\mathrm{H}_{2} \mathrm{O}}<9 \times 10^{25} \mathrm{~s}^{-1}$ (Stern et al. 1998) was approximated for $3 \mathrm{AU}$ which is almost two orders of magnitude higher than the production rates of the other species. The water density in a distance of $r=5 \mathrm{~km}$ is then estimated to be $\rho_{\mathrm{H}_{2} \mathrm{O}}<1.2 \times 10^{-11} \mathrm{~kg} / \mathrm{m}^{3}$, a factor of thirty higher than the density of the other species.

The interaction of the outflowing gas with the spacecraft having a maximal cross sectional area of $A=70 \mathrm{~m}^{2}$ and a mass of $m=1800 \mathrm{~kg}$ will result in a radial acceleration of the spacecraft by

$a=\frac{1}{2} C_{\mathrm{D}} \frac{A}{m} \rho v^{2}$ 


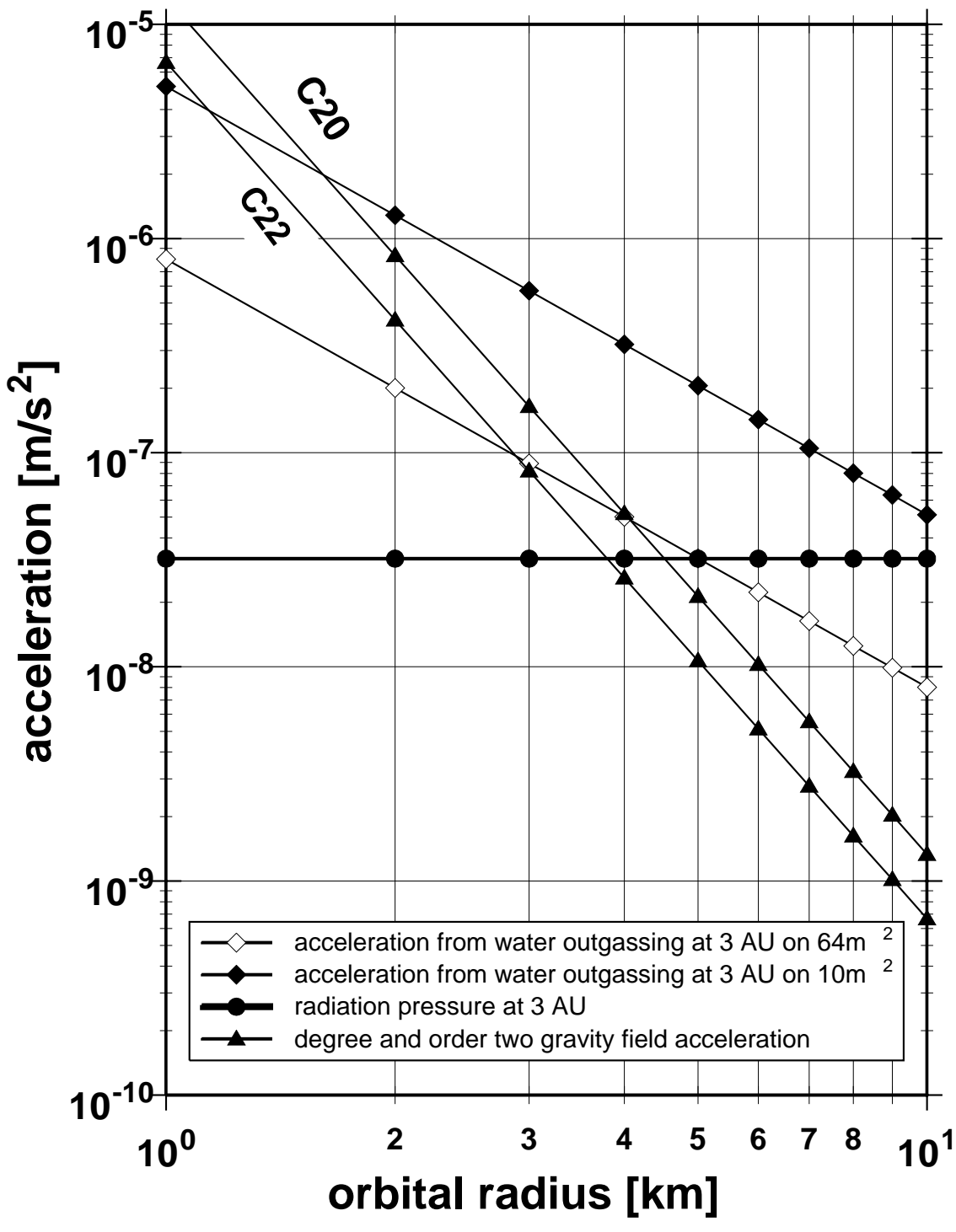

Fig. 7. Comparison of the second degree and order gravitational acceleration amplitudes with other nongravitational forces acting on the spacecraft: gas mass flux at $3 \mathrm{AU}$ (Schulz \& Schwehm 1999) and water mass flux at $3 \mathrm{AU}$ (Stern et al. 1998), radiation pressure at $3 \mathrm{AU}$ and 1.5 AU. The radiation pressure acceleration (circles) exceeds the gravitational acceleration outside of $4 \mathrm{~km}$ distance to the nucleus but can be predicted and considered in the analysis to a large extent. The mass flux interaction from the outflowing gas is the most problematic. At $3 \mathrm{AU}$ and at $5 \mathrm{~km}$ distance to the nucleus, the radial mass flux acceleration (open diamonds) is only a factor of two smaller and may even exceed the low degree and order gravitational acceleration at some location in orbit. if the mass flow is perpendicular to the full extent of the spacecraft cross sectional area and which is in the order of

$a_{\text {gas }}(R=3 \mathrm{AU}, r=5 \mathrm{~km})<7.4 \times 10^{-9} \mathrm{~m} / \mathrm{s}^{2}$

$a_{\mathrm{H}_{2} \mathrm{O}}(R=3 \mathrm{AU}, r=5 \mathrm{~km})<2.2 \times 10^{-7} \mathrm{~m} / \mathrm{s}^{2}$.

Specific spacecraft orbits have to be selected in order to reduce the perturbing effects from outgassing. The terminator orbit would present the smallest cross sectional area toward the mass flow. Choosing $A=10 \mathrm{~m}^{2}$ as an average in this orbit, the radial accelerations would be reduced to

$a_{\text {gas }}(R=3 \mathrm{AU}, r=5 \mathrm{~km})<1.0 \times 10^{-9} \mathrm{~m} / \mathrm{s}^{2}$

$a_{\mathrm{H}_{2} \mathrm{O}}(R=3 \mathrm{AU}, r=5 \mathrm{~km})<3.3 \times 10^{-8} \mathrm{~m} / \mathrm{s}^{2}$.

Water outgassing at $3 \mathrm{AU}$ will generate forces onto the spacecraft in the same order of magnitude as the gravity field at the same distance.

In comparison, with the onset of major cometary activity, the water production rate increased by one order of magnitude at 2.5 AU (Schulz \& Schwehm 1999) which yields

$a_{\mathrm{H}_{2} \mathrm{O}}(R=2.5 \mathrm{AU}, r=5 \mathrm{~km})<2.1 \times 10^{-7} \mathrm{~m} / \mathrm{s}^{2}$.

In this case the accelerations from outgassing are much larger than those from the gravity field (Fig. 7) and the derivation of the gravity field will not be feasible.

Radiation pressure is another non-gravitational force constantly acting on the spacecraft in anti-solar direction and is described by

$a=\frac{S_{0} A}{m c}\left(\frac{R_{0}}{R}\right)^{2} Q_{\mathrm{pr}}$

where $S_{0}=1366 \mathrm{~W} / \mathrm{m}^{2}$ is the solar constant at $R_{0}=$ $1 \mathrm{AU}, R$ is the heliocentric distance and $Q_{\mathrm{pr}}=1.21$ is the absorption and reflection coefficient of the solar cell array (Montenbruck \& Gill 2000). The radiation pressure yields a magnitude of

$a_{\mathrm{rad}}=3.2 \times 10^{-8} \mathrm{~m} / \mathrm{s}^{2}$ 
at $R=3 \mathrm{AU}$ which is again in the order of magnitude of the contributions from $C_{20}$ and $C_{22}$ at $5 \mathrm{~km}$ distance and overcomes even the outgassing accelerations for distances larger than $2 \mathrm{~km}$.

Solar radiation pressure may cause the orbiter to either crash into the nucleus or get ejected from a bound orbit in a reasonably short time span. As shown by Scheeres et al. (1998), this can be avoided by choosing special orbits. At distances from the nucleus where the solar radiation pressure is the dominant perturbation, the most interesting orbits are perhaps those perpendicular to the sun-comet direction (terminator orbit). The eccentricities of these orbits depend only on the mass of the nucleus, the distance from the Sun and the semi-major axes.

\section{Discussion and conclusion}

The radial gravitational acceleration amplitudes (9) are modulated by

$$
\begin{array}{r}
P_{20}(\cos \theta(t)) \\
P_{22}(\cos \theta(t)) \cos 2 \phi(t)
\end{array}
$$

where the cometary colatitude $\theta(t)$ and longitude $\phi(t)$ of the radius vector are functions of time. An important operational parameter is the required ground station tracking time to resolve the contribution from the low degree and order gravity coefficients not only from the background Doppler noise but also to cover the gravitational wavelengths with a sufficient number of Doppler measurements. The planned Doppler integration time of $600 \mathrm{~s}$ is driven by the need to reduce the background noise in order to resolve the forces acting on the spacecraft. A significant longer tracking time will be required for the derivation of the low degree and order gravity harmonics.

Derivation of degree two, order zero is determined by the revolution of the spacecraft in its polar orbit about the center of mass. At $5 \mathrm{~km}$ distance the revolution period is about 100 hours (Fig. 1) covering two wavelengths at degree two, order zero with 600 points of observations.

Boehnhardt et al. (2001), Lamy et al. (1998) and Meech et al. (1997) report a rotation period of the nucleus which is best compatible with a period of 5 to 7 hours. Degree and order two requires a full revolution period of 100 hours to cover the latitude dependence while the longitude dependence is covered within 2.5 to 3.5 hours at a certain latitude band.

In order to verify the measurements, a global gravity mapping might therefore be concluded in the relatively short time frame of two weeks in a low polar orbit within $5 \mathrm{~km}$ distance. Furthermore, a continuous tracking coverage by the ground stations is required for the time the spacecraft is not occulted by the nucleus.

Attempts will be made to apply solutions of ellipsoidal harmonics as described by Garmier \& Barriot (2001) and as demonstrated at Eros.

The determination of the harmonics of the gravity field would probably only be feasible if a gravity mapping campaign takes place at heliocentric distances well beyond $3 \mathrm{AU}$ when the comet is not very active and is best performed at heliocentric distances between 3.5 AU and $3.25 \mathrm{AU}$ before or at the onset of activity.

The comet will be near solar opposition at this time (mid 2012), thereby minimizing the effect of the interplanetary medium on the radio wave propagation. On the other hand, the constellation of opposition is also a disadvantage. A terminator orbit would be located in the plane-of-sky without inducing a Doppler velocity component along the line-of-sight. A solution would be an orbit inclined to the line-of-sight, inducing the highest Doppler component at the visible night sight of the comet and not considering the trajectory arc at the day sight.

From a pure mission point of view, considering the strong non-gravitational forces acting on the spacecraft, already the save operation and navigation of Rosetta will be very challenging in particular when approaching perihelion.

Acknowledgements. The RSI experiment is funded by the Deutsches Zentrum für Luft- und Raumfahrt (DLR), Bonn, Germany, under grants 50QP9709 and 50QP9717, by Norges forskningsraad, Norway, under project No. 136028/431, and by the National Aeronautics and Space Administration (NASA), Washington, USA.

\section{References}

Bird, M. K. 1982, Space Sci. Rev., 33, 99

Boehnhardt, H., Babion, J., \& West, R. M. 1997, A\&A, 320, 642

Boehnhardt, H., Delahodde, C., Sekiguchi, T., et al. 2001, VLT Kueyen observations of ROSETTA Target Comet 46P/Wirtanen near Aphelion, A\&A, submitted

Deprit, A. 1969, Celest. Mech., 1, 12

Drechsel, H., Bär, A., \& Boehnhardt, H. 2000, ESTEC study - Observations and modelling of large-scale dust environment in comets in preparation of the ROSETTA mission, ESTEC scientific research contract report - June 2000, Bamberg

Garmier, R., \& Barriot, J.-P. 2001, Ellipsoidal harmonic expansion of the gravitational potential: Theory and Application, Cel. Mech. Dyn. Astron., in press

Gill, E., Montenbruck, O., \& Pätzold, M. 1996, Perturbation forces acting on the Rosetta spacecraft in a close orbit around comet P/Wirtanen, Paper AAS 96-150, AAS/AIAA Space Flight Mechanics Meeting, Austin, Texas, 12-15. Feb.

Greenberg, J. M., \& Hage, J. I. 1990, ApJ, 361, 260

Keller, H. U., et al. 1986, A\&A, 187, 807

Krankowsky, D. P., Lämmerzahl, J., Woweries, et al. 1986, Nature, 321, 326

Lamy, P. L., Toth, I., Jorda, L., et al. 1998, A\&A, 335, L25

Meech, K. J., Bauer, L. M., \& Hainaut, O. R. 1997, A\&A, 326, 1268

Milani, A., Nobili, A. M., \& Farinella, P. 1987, Nongravitational perturbations and satellite geodesy (Adam Hilger, Bristol)

Miller, J. K., Williams, B. G., Bollman, W. E., et al. 1995, J. Astronaut. Sci., 43, 453 
Montenbruck, O., \& Gill, E. 2000, Satellite Orbits (Springer, Berlin, Heidelberg, New York)

Olsen, O. 2000, An analytical study of orbits around rotating nonsymmetric objects, Master of Science Thesis, University of Oslo

Pätzold, M., et al. 2001, Rosetta Radio Science Investigations, ESA-Special Publication, in press

Rickman, H. 1989, Space Res., 9(3), 59

Rickman, H., Kamel, L., Festou, M. C., \& Froeschle, C. 1987, in Diversity and Similarity of Comets, ed. E. J. Rolfe, \& B. Battrick, ESA SP-278, 471
Scheeres, D. J., Ostro, S. J., Hudson, R. S., \& Werner, R. A. 1995, Icarus 121, 67

Scheeres, D. J., Marzari, F., Tomasella, L., \& Vanzani, V. 1998, Planet. Space Sci., 46, 649

Schulz, R., \& Schwehm, G. 1999, Space Sci. Rev., 90, 321

Sekanina, Z., \& Yeomans, D. K. 1985, AJ., 90, 2335

Stern, S. A., Parker, J. W., Festou, M. C., et al. 1998, A\&A, 335, L30

Yeomans, D. K., Antreasian, P. G., Barriot, J.-P., et al. 2000, Science, 289, 2085 